\title{
Chemical Fixation Importance of Histologic Samples for Staining of Proliferated Bone During Implant Osseointegration
}

\author{
Teodora MARCU ${ }^{1}$, Adrian GAL ${ }^{2}$, Cristian MARTONOS ${ }^{2}$, Vasile RUS ${ }^{2}$, Aurel DAMIAN²*, Viorel MICLĂUȘ², \\ Augustin T. MIHAI ${ }^{3}$ \\ ${ }_{1}^{1}$ „Dunărea de jos” Universtity of Galați, Faculty of Medicine and Pharmacy, Department of Dental Medicine \\ ${ }^{2}$ University of Agricultural Sciences and Veterinary Medicine of Cluj-Napoca, Faculty of Veterinary \\ Medicine, Department of Preclinical Sciences \\ ${ }^{3}$ University of Medicine and Pharmacy „Carol Davila” Bucharest, Faculty of Dental Medicine \\ *corresponding author: damian56aurel@yahoo.com
}

Bulletin UASVM Veterinary Medicine 77(2)/2020

Print ISSN 1843-5270; Electronic ISSN 1843-5378

doi:10.15835/buasvmen-vm:2020.0034

\begin{abstract}
This study aimed to obtain as much and as accurate information as possible about the process of osseointegration of titanium implants, through histological processing. For this purpose, the combination of a mercury chloride fixator (Stieve mixture) and a complex staining method (Goldner's trichrome) was used, which can highlight the microscopic structures in several colors and shades. The Stieve mixture proved to have distinguished qualities both in the preservation of basophilic and acidophilic structures and especially in the chemical preparation of the substrate for the binding of each structural component with a specific acidophilic dye. On the substrate thus prepared, the acidophilic dyes used in the Goldner's trichrome staining highlighted the structural components in different colors and shades, offering the possibility of high precision assessment of the newly proliferated bone, in different stages of proliferation, consolidation and reshaping. Complex information was obtained, which allows the appreciation of osseointegration for endosseous implants and the amount of proliferated bone at the boneimplant interface, the stage of proliferation, consolidation and reshaping of the mature bone, the degree of fixation to deep bone structures. The possibility of complex assessment of the osseointegration process for endosseous implants at a given time is of great practical importance for correct assessment of the waiting time required for safe installation of prosthesis. The combination of Stieve fixator mixture with Goldner's trichrome staining method has proven to be very effective in histology, highlighting on excellent level the existing structural components of each stage, in which the osseointegration process goes through. A very important role in this combination was achieved by mercury chloride mixture, which prepared the substrate for specific reaction with dyes. Regarding the results obtained, we recommend this combination for histological investigations aimed on bone proliferation and repair.
\end{abstract}

Keywords: bone implant, chemical fixation, osseointegration

\section{Introduction}

In the process of fixing the samples, all of the vital processes must be stopped. This stopping process is mandatory in order to prepare the samples for histological examination. In order to evaluate some histophysiological or histopathological processes, it is imperative to choose the correct fixator (Mureșan et al., 1974). In order to examine a tissue or an organ the fixator must be chosen carefully so that the substance achieves the best method of fixation for the various staining methods which will follow.

A substance which allows the coagulation of proteins is represented by mercuric chloride. 
Mercury chloride is considered by many specialists as one of the best fixation agents (Gabe, 1968). The advantage of mercury chloride is represented by the fact that it chemically prepares the substrate for staining substances. After using mercury chloride-based fixators, they present a special brightness (Kiernan,1981; 1990; Suvarna et al., 2018). All of the chemical mechanisms by which mercury chloride achieves fixation of the tissues is not yet fully understood. It is known to react with some amines, amides, amino acids and sulfhydryl groups, with phosphate residues of nucleic acids and nucleic proteins, which is why it is used for high-quality nuclear preservation (Rolls, 2012). The theory is supported by other authors too, who claim that after fixation with mercury chloride nuclear and cytoplasmic staining is of high grade staining (Sanderson, 1994).

The tissue penetration is directly linked with the diffusion capacity of the fixator. The fastest penetration is achieved by alcohol and formalin, but the slowest is represented by glutaraldehyde. Mercury chloride has a somewhat intermediate penetrability between the two substances (Scarano et al., 2003).

Mercury chloride is rarely used as a fixator agent alone. More frequently is used as a base component in some fixation mixtures. After fixation with mercury chloride mixtures, the staining affinity of the tissues for both acidic and basic dyes is obviously enhanced. For these arguments, fixators containing mercury chloride are often preferred for trichrome staining methods (Baker, 1958, Geoffrey Rolls, 2012). One of the most used stains in complex bone tissue investigations is the Goldner's trichrome method, which manages to differentiate the osteoid and the primary plexiform bone from mineralized mature bone tissue (Gruber and Ingram, 2003). This differentiation is largely based on the fact that Goldner's trichrome is a selective staining technique for collagen fibres, giving them a specific colour (Gabe, 1968). Goldner's trichrome method is an alternative for Masson stain (1929), which is recommended after fixation with mercury chloride mixtures (Bancroft and Layton, 2019).

The Tricrom Goldner staining is one of the fewest methods which allows fine overview of the tissue and offers details regarding the stages of the processes which take place (Claudia Rentsch et al., 2014).
In order to obtain the most complete results from a qualitative point of view in assessing the osseointegration process of a titanium implant, we have chosen the experimental rabbit model (Pearce și col. 2007; Wancket, 2015). The objective of this study was to identify the efficiency of the mercury chloride fixation combined with tricrom Goldner staining on bone tissue samples.

\section{Materials and methods}

For this study, three 10-month-old male rabbits were used, in which titanium implants with a thread, with a diameter of $2 \mathrm{~mm}$ and a length of $5 \mathrm{~mm}$, were inserted in the middle portion of the femur. Pierce et al. (2007), states that the size of the implants used must not exceed more than $2 \mathrm{~mm}$ in diameter and $6 \mathrm{~mm}$ in length, meaning about half the size recommended for larger species (International Standard IS010993$6,1994)$. Four weeks after insertion, the rabbits were euthanised, moment when the portion of the femur containing the implant was sampled for histological investigations. The samples were fixed in the Stieve mixture for three days, then dehydrated with ethyl alcohol, clarified with butyl alcohol (1-Butanol) and submerged in paraffin. Sections with a thickness of 5 micrometres were cut and stained with Goldner's trichrome method. An Olympus BX41 microscope equipped with a digital camera for image capturing was used to examine the histological samples. The later processing of the histological images was done with Adobe Photoshop 2020 programme.

\section{Results and discussions}

Histological processing of the area in which the titanium implant was inserted provides complete information on the body tolerance degree in conjunction with the implant and implant integration in the host tissue. It offers information regarding soft peri-implantation in the supraperiosteal and subendosteal areas, and especially on the tissue which proliferated at the bone-implant interface. The periosteum and connective tissue in the immediate proximity of the suprasosseous portion of the implant appear without inflammatory reaction and with abundant structural details in the cellular and fibrillar components. From the internal part of the periosteum, a large number of osteoblasts are mobilized, which initiate and support bone 
proliferation in the upper part of the bone-implant interface (Figure 1). The situation is similar for the bone marrow, located in the medullary canal, that comes in contact with the portion of the implant, which exceeds the thickness of the femoral bone. There were no current signs of inflammation. The area in immediate proximity with the implant is occupied by a loose connective tissue, consisting of thin connective fibres and discrete tinctorial affinity, which proves that the tissue is very young. This tissue is continued, without any delimitation, by the bone marrow with a normal structure. From the endosteum, a large number of osteoblasts are mobilized, which contribute to the bone proliferation in the endosteal portion of the bone-implant interface.

On the proliferated bone, on the bone implant interface, all of the components could be observed, both cellular and bone matrix structures. In this relatively young bone, osteoblasts were present in large numbers, both on bone-implant interface and in the polymorphic trabeculae. The osteoclasts which are present in Figure 3 take part in the lysis of the bone fragments which were formed after the threading. Osteocytes are still present in the gaps of the matrix and some of them are surrounded by the osteoid (Figures 2, 3). The bone matrix has a polymorphic appearance. The staining allows the evaluation of the fresh proliferated bone with precise differentiation of all stages that it goes through, from osteoid to remodelled bone, from secondary to lamellar bone. Bone lamellae can be appreciated from the stage of primary lamella to the consolidated lamellae, with precise evaluation of their orientation, either parallel (Figure 4) or concentric (Figure 5), depending on the structures in which they are found. The non-viable bone could be observed on the borderline between the newly proliferated bone and the deeper bone structures. The non-viable bone will be gradually replaced by the newly proliferated bone which will ensure the strong binding of the bone, on the interface with the deeper bone structures (Figure 6).

The utility of these histological investigations, in evaluation of implant osseointegration, is supported by specialists in the field which claim that, although it is a complex and laborious method, it provides excellent details on the events accompanying osteointegration, depending on each stage in which this laborious process goes through (Rentsch et al., 2014). No other method of investigation provides as many detailed information, and when it follows X-rays and CT scans, new and modern results are achieved.

During osseointegration, the process of bone proliferation takes place, bone regeneration and bone reshaping begins. There are several stages of bone proliferation and consolidation processes at the same time. The evaluation of bone matrix regarding the structure and consolidation status provides information necessary for the osseointegration. In other words, for the correct assessment of osseointegration, we must be able to evaluate the amount of proliferated bone at the bone-implant interface. In order to highlight the ongoing processes, which involve many stages and aspects, an appropriate technique is needed to differentiate the components according to their structure and degree of maturation. One of the best methods, from this point of view, is Goldner's trichrome staining, which allows the staining of the connective tissue, depending on the consolidation stage of the fibrillar component in the newly proliferated bone, but also in consolidated one. The staining of connective tissue is so distinct, that some authors believe that this method could be used even for quantitative measurements (Rentsch, et al., 2014). Information on how this staining method manages to deal with so many chromatic details, can be found in literature. Thus, Gabe (1968) states that, the appearance is given by use of several acid dyes between which there is competition for binding with the substrate. This competition depends on the particles size, diffusion coefficients and especially the intervention of phosphomolybdenic or phosphotungstic acid, which are used as differentiators to remove a dye from certain structures so that they can couple with another dye.

In order for all these processes to run in the best conditions, the substrate must be very well prepared chemically for binding with specific dyes. One of the most effective substances in chemical fixation of bone components and enhancing their tinctorial affinity is mercury chloride. Used alone, it can harden the sample, especially if the fixing period is prolonged, with the appearance of difficulties in sectioning (Eltoum et al., 2001, Carson, 2007). To avoid hardening of the samples, mercuric chloride is used as a basic component in fixation mixtures containing substances that prevent this shortcoming, such as acetic acid, 


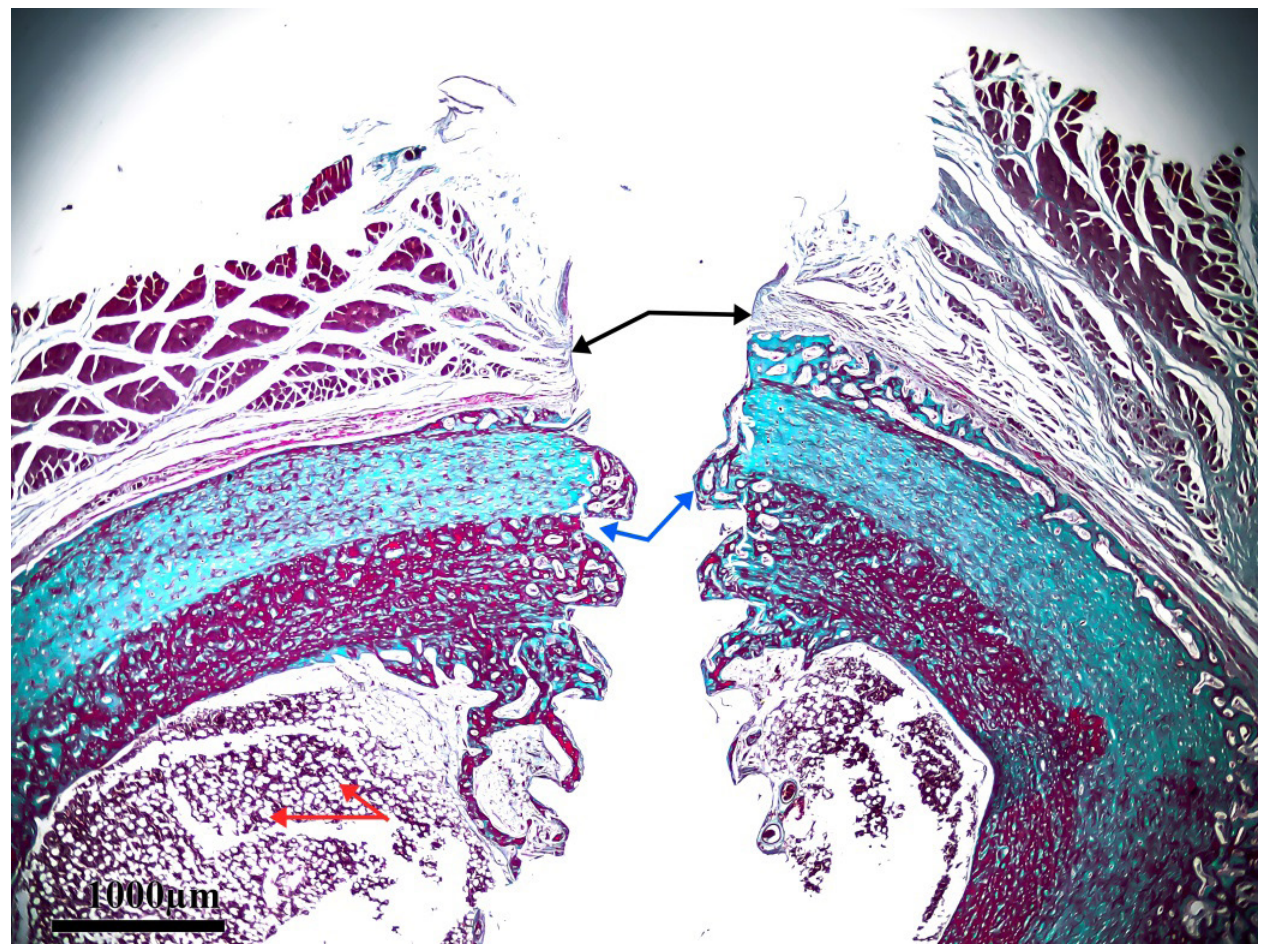

Figure 1. Implant after 4 weeks in a male - interface, soft and hard tissues (TG): black arrows-extraperiosteal soft tissues; blue arrows - bone-implant interface; red arrow - marrow from the medullary canal.

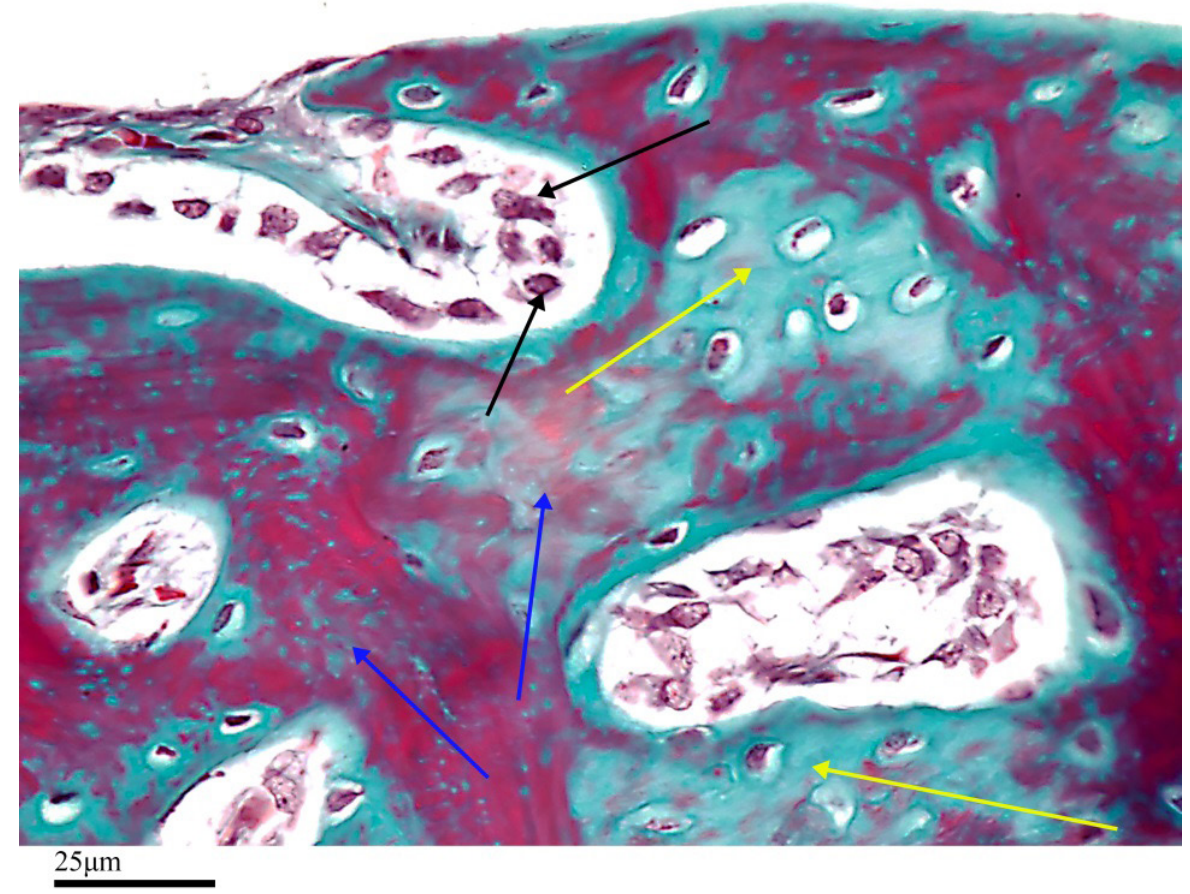

Figure 2. Bone-implant interface (TG): black arrows - osteoblasts; yellow arrows - osteoid; blue arrows - primary bone in various stages of consolidation. 


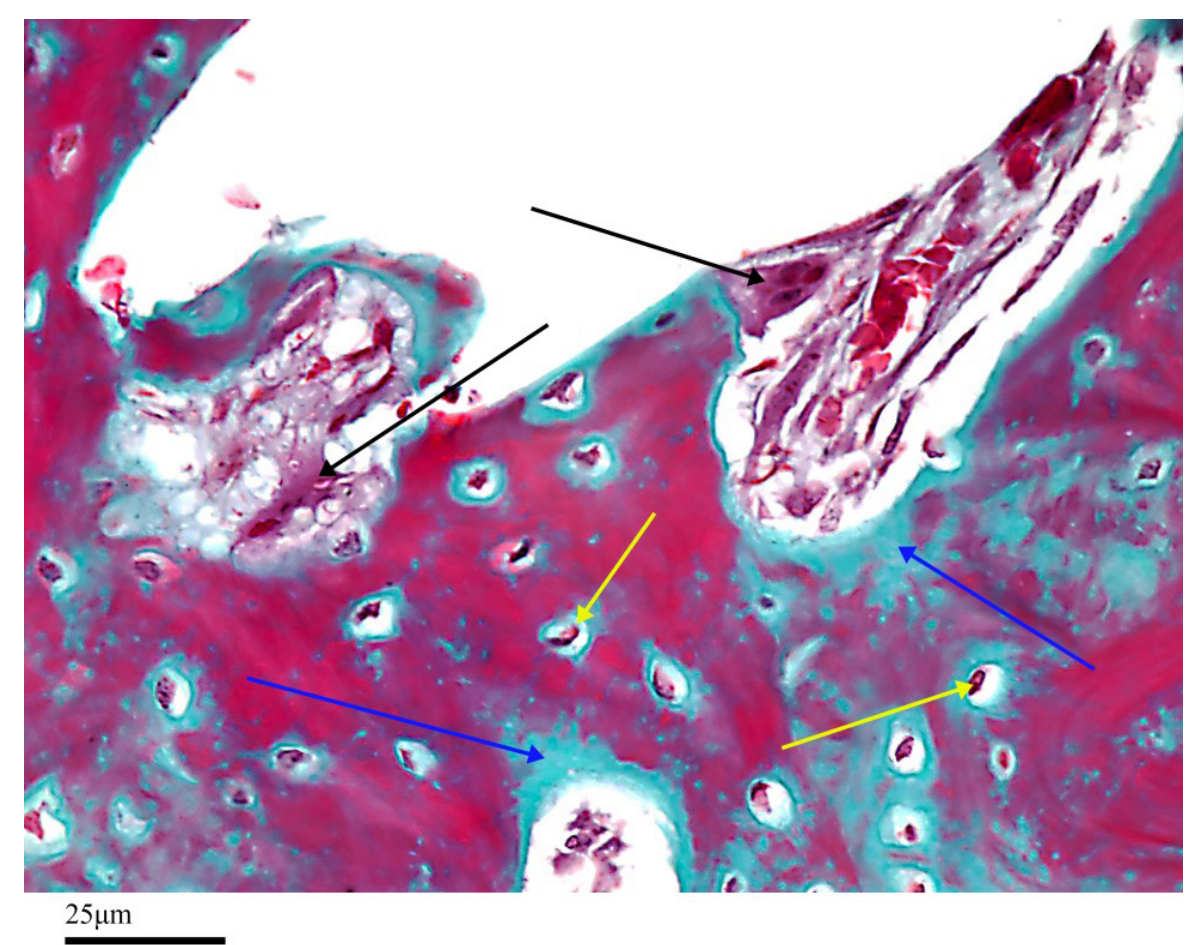

Figure 3. Bone-implant interface (TG): black arrows - osteoclasts; yellow arrows - osteocytes; blue arrows - osteoid.

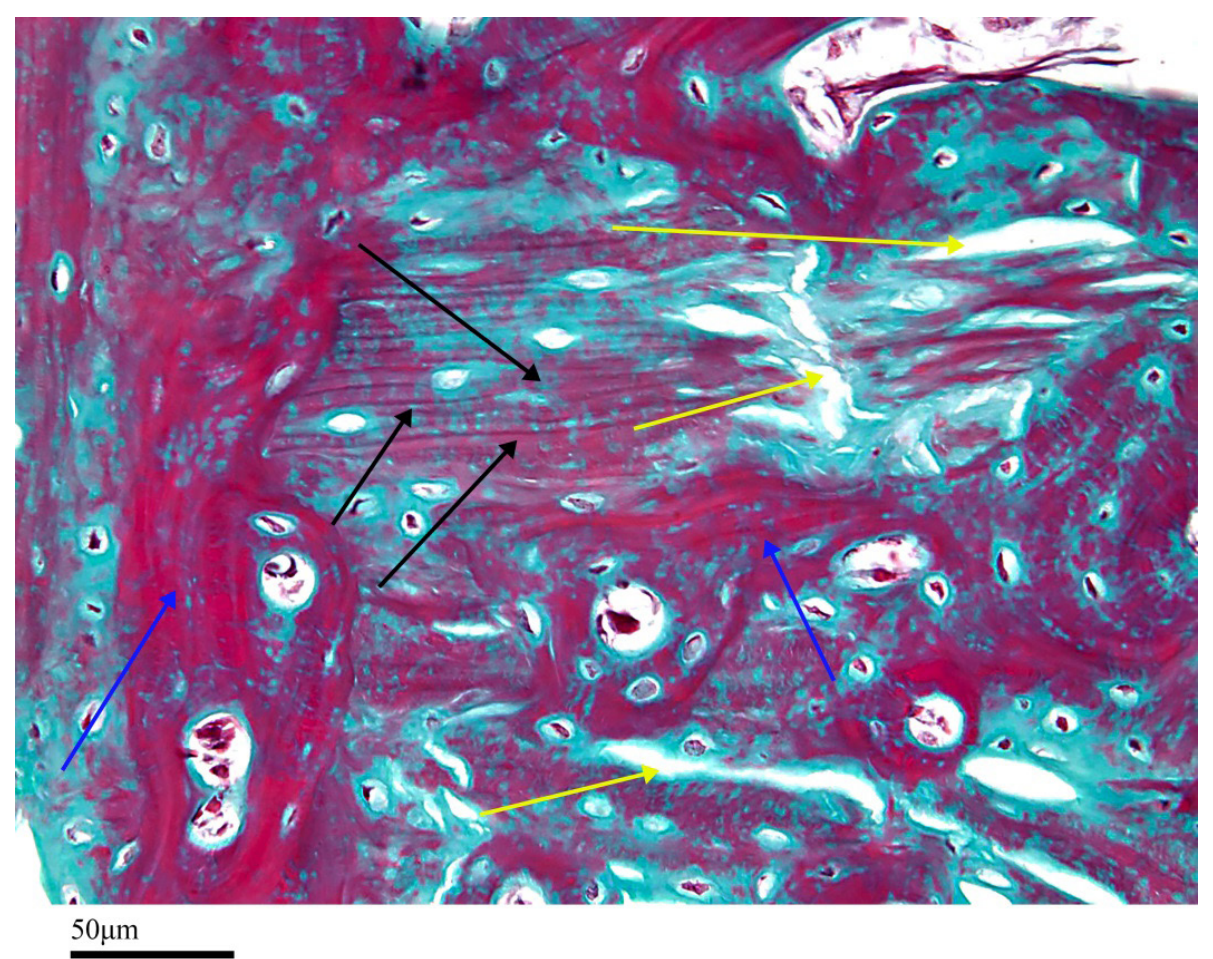

Figure 4. Bone-implant interface (TG): black arrows - parallel bone lamellae; yellow arrows - resorption canals; blue arrows - primary bone in various stages of consolidation. 


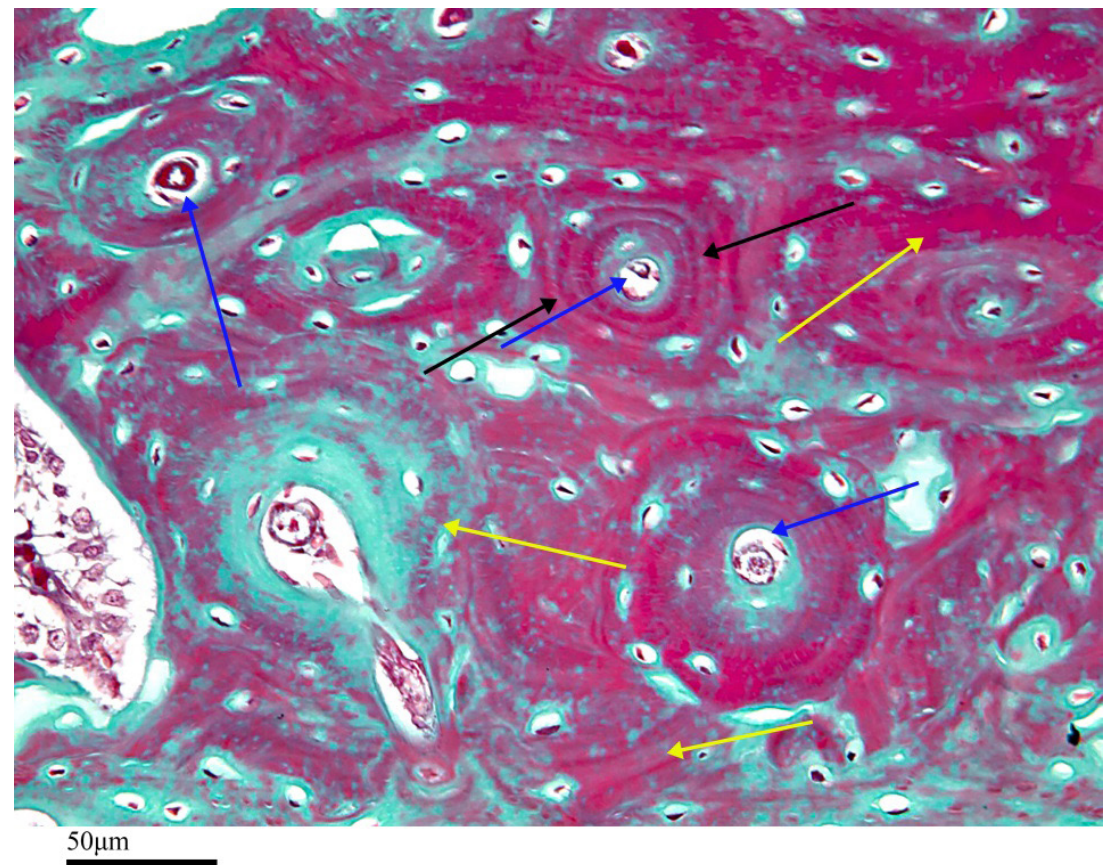

Figure 5. Bone-implant interface (TG):black arrows - concentric bone lamellae in the osteon; yellow arrows - primary bone in various stages of consolidation; blue arrows - Haversian canals.

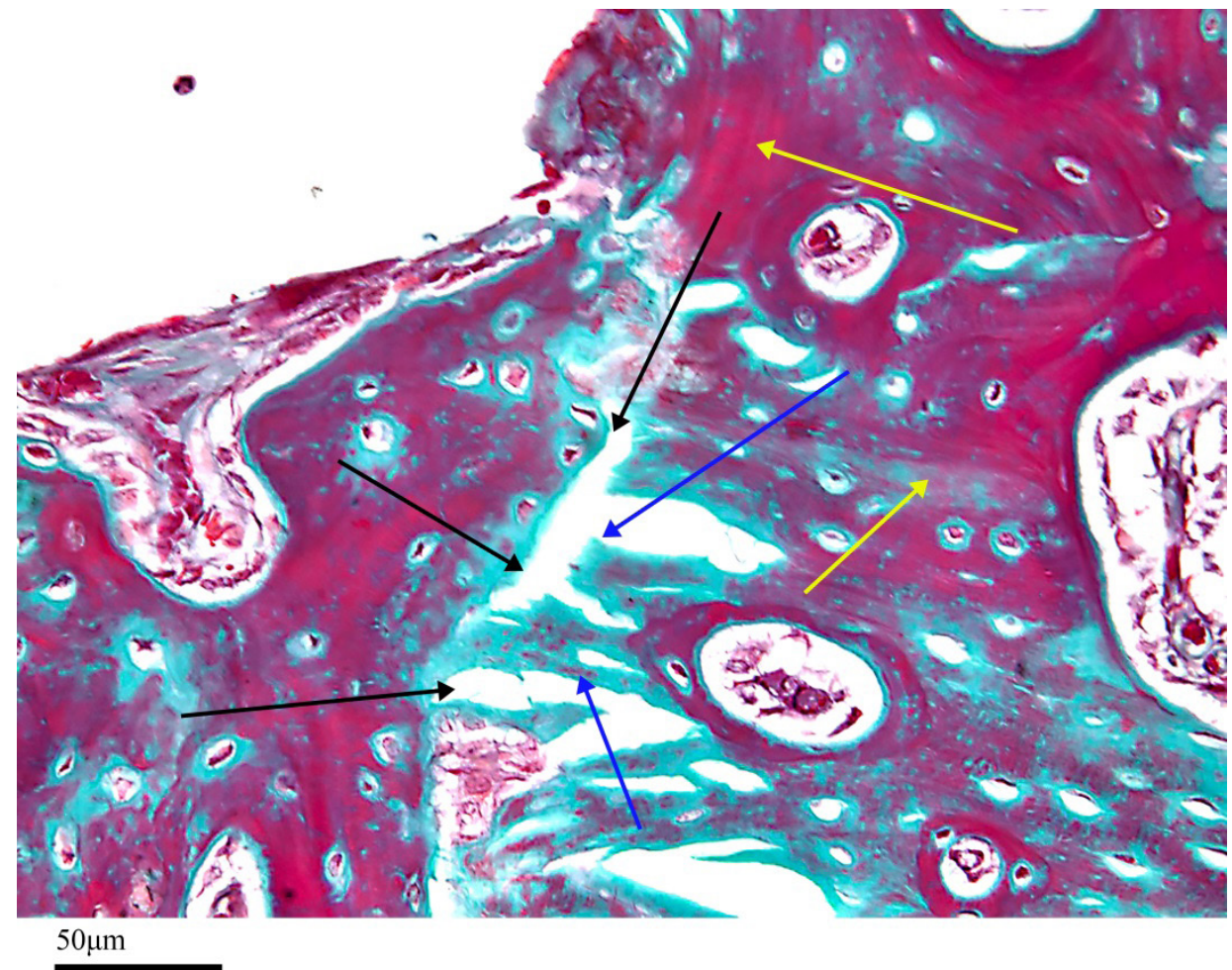

Figure 6. Bone-implant interface (TG): black arrows - the junction area with deep bone structures; yellow arrows - primary bone in various stages of consolidation; blue arrows - unviable bone. 
formaldehyde, potassium dichromate etc. (Culling, 1963). One of such fixation mixtures, is the one we used, the Stieve mixture, which contains mercuric chloride, acetic acid and formalin. This fixating agent, in addition to binding very well to both acidophilic and basophilic structures, does not harden the samples so they can be easily cut, without the appearance of cracks or ruptures in the tissues. Moreover, this fixating agent has a certain tolerance related to the time period in which the samples stay in the fastener. If the optimal fixing time is exceeded, the hardening and chemical damage of the samples do not occur.

The results obtained in this study show that the chosen method, ensured a very good processing and chemical preparation of the samples. The acidophilic structures existing in the proliferated bone and at bone-implant interface were highlighted in different colours and shades. This feature offers the possibility of high precision evaluation of newly proliferated bone, in various stages of proliferation, consolidation and reshaping. This information is very useful for accurate assessment of the stage reached in the osseointegration process. Consequently, we can assess: the amount of proliferated bone at the bone-implant interface, the stage of proliferation, consolidation and reshaping towards mature bone, the degree of anchoring in correlation with deep bone structures. These aspects are very important for the correct appreciation of required time for a proper prosthesis mounting.

\section{Conclusion}

The fixating agent we used has been proven to possess special qualities, both in the preservation of basophilic and acidophilic structures and especially in the chemical preparation of the substrate, for binding of each structural component with a specific acidophilic dye.

The specific binding of acidophilic dyes to the structural components that they highlight in different colours and shades, offers the possibility of high precision assessment of new bone matrix, in different proliferation stages, consolidation and reshaping.

The combination of Stieve fixator with Goldner's trichrome staining method, offers excellent details about osseointegration, more precisely the amount of bone proliferated at the bone-implant interface, the stage of proliferation, consolidation and reshaping to mature bone, the merging degree with the deep bone structures.

Regarding the mounting of the prosthesis, when it comes down to the waiting period, at the moment of examination, it is very important to take into account the stage of the osseointegration process.

Acknowledgments. This research did not receive any specific grant from funding agencies in the public, commercial, or not-for-profit sectors.

\section{References}

1. Baker JR (1958). Principles of biological microtechnique Methuen, London, UK.

2. Bancroft JD, Layton C (2019). Connective and other mesenchymal tissues with their stains In: Bancroft's Theory and Practice of Histological Techniques (Eighth Edition) , p. 153-175

3. Carson FL (2007). Histotechnology. 2nd ed. Chicago: ASCP Press.

4. Culling CFA (1963). Handbook Histopathological Techniques, second edition, London, Butterworths

5. Eltoum I, Fredenburgh J, Myers RB, Grizzle WE (2001). Introduction to the theory and practice of fixation of tissues. J Histotechnol;24;173 -190.

6. Gabe M (1968). Techniques Histologiques, Masson Et $\mathrm{C}^{\mathrm{ie}}$, Editeurs, Paris

7. Gruber HE, Ingram JA (2003). Basic Staining and Histochemical Techniques and Immunohistochemical Localizations Using Bone Sections In: Handbook of Histology Methods for Bone and Cartilage Edited by: Y. H. An and K. L. Martin (C) Humana Press Inc., Totowa, p.181

8. Kiernan JA (1981). Histological \& Histochemical Metods, Teory and Practice, (1 st ed.), Oxford, Pergamon Press

9. Kiernan JA (1990). Histological \& Histochemical Metods, Teory and Practice, Second Edition, Oxford, Pergamon Press

10. Mureșan E, Gaboreanu M, Bogdan AT, Baba AI (1974). Tehnici de histologie normală și patologică, Editura Ceres, București.

11. Pearce AI, Richards RG, Milz S, Schneider E, Pearce SG (2007). Animal models for implant biomaterial research in bone: a review. European Cells and Materials; 13, p.1 - 10.

12. Rentsch C, Schneiders W, Manthey S, Rentsch B, Rammelt S, (2014). Comprehensive histological evaluation of bone implants, Biomatter, 4:1, e27993, DOI: 10.4161/ biom. 27993

13. Rolls G (2012). Fixation and Fixatives (3) - Fixing Agents Other than the Common Aldehydes

14. Sanderson JB (1994). Biological microtechnique, Bios Scientific Publication, Oxford.

15. Scarano A, Iezzi G, Piattelli A (2003). Common Fixatives In Hard-Tissue Histology, In: Handbook of Histology 
Methods for Bone and Cartilage Edited by:Y. H. An and K. L. Martin (C) Humana Press Inc., Totowa, p. 159

16. Suvarna K, Layton C, Bancroft JD (2018). Bancroft's Theory and Practice of Histological Techniques 8th Edition, Elsevier
17. Wancket LM (2015). Animal Models for Evaluation of Bone Implants and Devices: Comparative Bone Structure and Common Model Use, Veterinary Pathology 52(5), p. 842-850. 